\title{
Measurement-based Simulation of Underwater Optical Networks
}

\author{
Filippo Campagnaro ${ }^{\S \star}$, Matteo Calore ${ }^{\S}$, Paolo Casari ${ }^{\ddagger}$, Violeta Sanjuan Calzado ${ }^{\sharp}$, \\ Giacomo Cupertino $^{\circ}$, Claudio Moriconi ${ }^{\circ}$, Michele Zorzi ${ }^{\S}$ \\ ${ }^{\S}$ Department of Information Engineering, University of Padova, via Gradenigo 6/B, 35131 Padova, Italy \\ ${ }^{\ddagger}$ IMDEA Networks Institute, Madrid, Spain \\ \#NATO STO Centre for Maritime Research and Experimentation, viale San Bartolomeo 400, La Spezia, Italy \\ ${ }^{\circ}$ ENEA Centro della Casaccia, via Anguillarese 301, Roma, Italy \\ ${ }^{\star}$ Corresponding author. Email: campagn1@dei.unipd.it
}

\begin{abstract}
The increasing interest in the application of optical communications to underwater communications and networks calls for reliable simulation models, that allow a system designer to realistically assess the performance of optical systems before actual deployment in water. In this paper, we present an optical channel model that is based on samples of the total attenuation coefficient and of solar light irradiance taken during the NATO STO CMRE ALOMEX'15 scientific cruise, in different water conditions. The data set includes both strong and mild solar illumination conditions, as well as a number of different settings for the light absorption and scattering coefficients.

We implement the model as part of the DESERT Underwater network simulator, and employ it to show that the throughput of underwater optical links depends not only on the distance between the transmitter and the receiver, but also on the depth at which the devices are deployed. The corresponding insight is empirically validated in dry tests and lake experiments using a proof-of-concept optical modem, and helped drive the next stage of the modem development.
\end{abstract}

Index Terms-Underwater optical communications; multimodal underwater networks; NATO CMRE; ALOMEX; channel models; simulation; field measurements; DESERT Underwater

\section{INTRODUCTION AND RELATED WORK}

Underwater optical communications are currently concentrating increasing interest. Many recently developed optical modems are commercially available (e.g., [1]-[3]) and used in deep water Oil\&Gas $(\mathrm{O} \& \mathrm{G})$ assets. However, most of these systems do not exist as commercial off-the-shelf (COTS) products, but rather as customized implementations which typically incur an expensive development phase. Several universities and research institutes proposed low-cost underwater optical proof-of-concept (PoC) modems (e.g. [4], [5]); however, none of them has been made commercially available, or reportedly employed in a complex networking experiment so far.

Before a real deployment of an underwater optical network, a preliminary system evaluation involving system-level analysis and simulations is required. During the simulations, it is essential to consider realistic scenarios, based not only on theoretical models, but also on field measurements with real hardware. Only in this way can the simulation results provide the first evaluation of a real system with high accuracy. Several theoretical models for underwater optical communications, either analytical or based on Monte-Carlo approaches, have already been addressed in the literature [6], [7]. However, they typically do not address time-varying optical channels or depth-varying ambient light noise, and they are typically based on physical models that require complex computations [8], [9]. The Woods Hole Oceanographic Institution has performed several sea trials to evaluate their optical modem under different water conditions. This includes deep water scenarios [10], shallow water scenarios at night [11] as well as in daytime conditions [12]. During the design phase in [11], they have also performed some preliminary simulations of optical transmissions. In [13], the authors present their hybrid optical and acoustic communications system, and employ the system in data-muling and vehicle control operations. Although these results are very interesting and demonstrate the effectiveness of the device, the authors have not released any underwater acoustic and optical network simulator based on the performance figures obtained this way.

In this paper, we aim to propose a solution to address the need for an optical communication model that is amenable to network simulations. Specifically, we propose the first underwater optical network simulator based on real measurements of optical propagation in ocean waters of different types. Such parameters were measured during the ALOMEX'15 research cruise, organized by the NATO STO Centre of Marine Research and Experimentation (CMRE). The ALOMEX'15 campaign spanned 13 days and covered a total route of about $2800 \mathrm{~km}$, starting from Cartagena, Spain, down to the West Saharian sea, and with final destination Las Palmas de Gran Canaria, Spain (see Fig. 1). During the cruise, the research vessel R/V Alliance has been navigated to 39 different stations to collect measurements. In each site, the measurements included the down-welling solar light irradiance, the optical attenuation and absorption coefficients and the water temperature throughout the water column. The measurements have been explicitly taken at different locations and times of day, in order to encompass diverse environmental conditions and water properties.

All measurements have been processed and embedded in the multi-modal acoustic and optical DESERT Underwater 


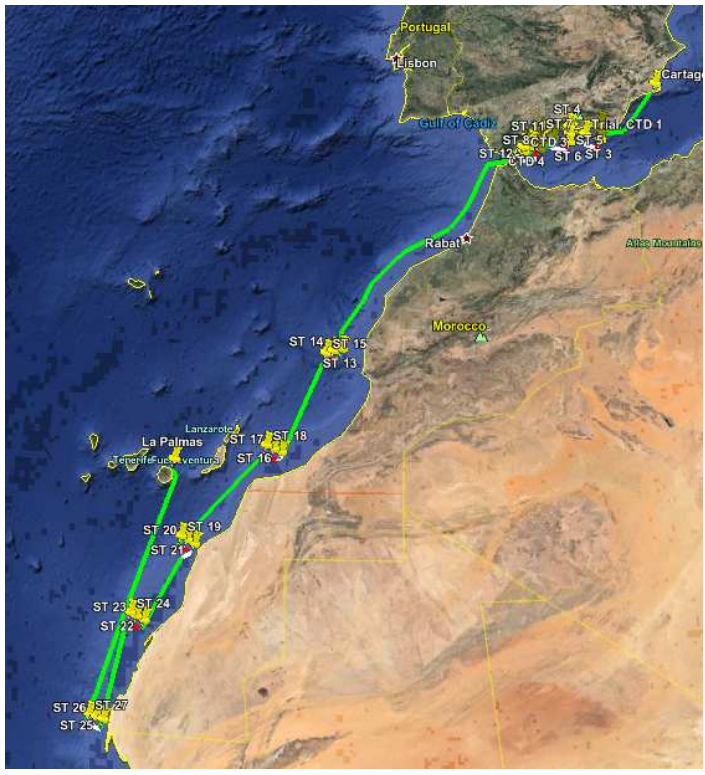

Figure 1. Cruise plan of the NATO ALOMEX'15 research cruise.

Network Simulator [14] in the form of lookup-tables (LUTs), which are used to characterize the optical channel. An example of such data is reported in Fig. 2, that shows the absorption rate $a$, the scattering rate $b$ and the total attenuation rate $c$. The latter quantity models the light energy loss due to propagation as a function of distance, and depends on depth. This data is reported for stations 2 and 7 in Fig. 1, which are representative of very different water types. The simulator also supports the connection to both acoustic and optical real underwater modems, such as all the EvoLogics S2C acoustic modems [15] and the ENEA optical modem prototype, to perform multimodal sea trials, and thus also serves as a network emulator or proof-of-design system for new technologies [16].

Our contribution in this paper is three-fold. First, we present the implementation details of the optical simulator, by centering our study on a sample scenario, as described in Section V. Second, we employ the model to carry out a simple simulation study using the open access DESERT Underwater network simulator. This study makes it possible to assess the impact of different ambient light and water conditions on the performance of optical links, based on real data. Finally, we perform preliminary experiments using the $\mathrm{PoC}$ optical modem of the Italian ENEA research institute [4] both in dark water and in very shallow water during a sunny day, and measured the packet delivery ratio in both conditions. The preliminary optical communication simulations and the results of the optical modem tests in sunny shallow waters served as an estimate of the ENEA modem performance in the presence of a solar light filter, and motivated the development of such filter for the next version the modem.

In the next section, we describe the capability of the DESERT Underwater framework to simulate multi-physical layer optical and acoustic networks.
Table I

OPTICAL PROPERTIES: NOTATION AND MEANING

$\begin{array}{lll}\text { Notation } & \text { Meaning } & \text { Value } \\ P_{0} & \text { Source-radiated optical power } & 100 \mathrm{~W} \\ B & \text { Signal bandwidth } & 100 \mathrm{kHz} \\ A_{t} & \text { Transmitter area } & 10 \mathrm{~mm}^{2} \\ \theta & \text { Transmitter's semi-aperture } & 1 \mathrm{rad} \\ A_{r} & \text { Receiver area } & 1.1 \mathrm{~mm}^{2} \\ S & \text { Receiver sensitivity } & 0.26 \mathrm{~A} / \mathrm{W} \\ R & \text { Receiver's shunt resistance } & 1.43 \mathrm{G} \Omega \\ I_{d} & \text { Photodetector's dark current } & 1 \mathrm{nA} \\ q & \text { Elementary charge } & 1.610^{-19} \mathrm{C} \\ K & \text { Boltzmann constant } & 1.3810^{-23} \mathrm{JK}^{-1} \\ T & \text { Temperature } & \text { From measurements } \\ E_{0} & \text { Scalar irradiance of the solar light } & \text { From measurements } \\ a & \text { Absorption rate } & \text { From measurements } \\ b & \text { Scattering rate } & \text { From measurements } \\ c & \text { Total attenuation rate } & \text { From measurements }\end{array}$

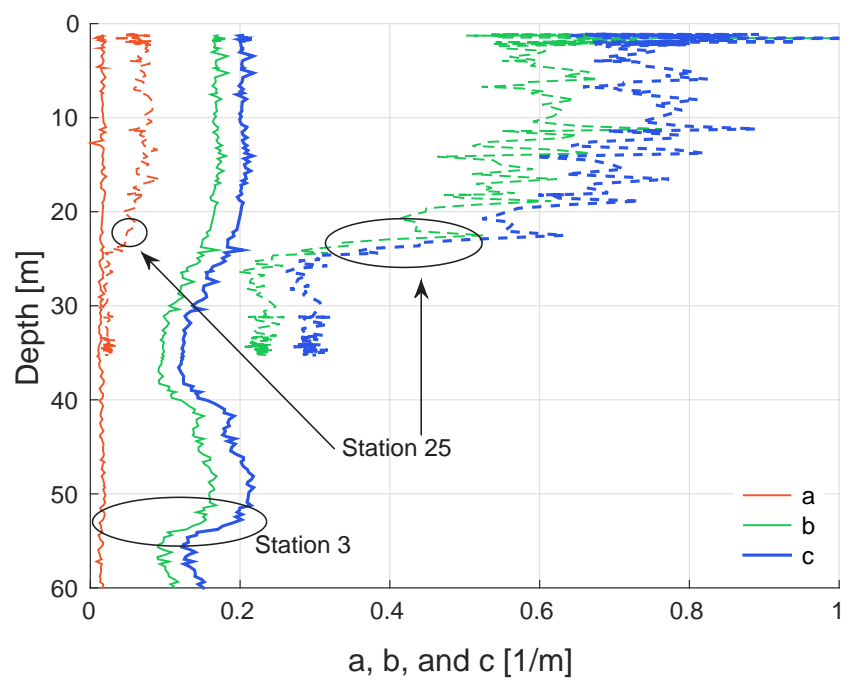

Figure 2. Measured light absorption ( $a$, red) scattering $(b$, green) and total attenuation ( $c$, blue) coefficients for stations 3 and 25 in Fig. 1.

\section{Measurement-BASED opticAl Simulator}

We start from the implementation of a measurement-based optical communication simulator. This tool has been included in the DESERT Underwater framework [14], a state of the art underwater multimodal acoustic and optical network simulator and emulator. The optical transmission model is described in [6]: we extended such model in order to include the most important variable optical parameters of the channel, namely, attenuation coefficient $c$, scalar irradiance of the solar light $E_{0}$ and temperature $t$, as lookup tables (LUTs). In the following subsections we describe how such LUTs have been obtained from the raw data acquired during the ALOMEX'15 NATO cruise, and how they have been integrated in the optical propagation simulator. In Table I we list the optical parameters employed in the optical SNR formula. 


\section{A. Attenuation coefficients and received power}

The optical absorption $a$ and attenuation coefficients $c$ of the water were measured using a Wet Labs Conductivity, Temperature and Depth (CTD) system equipped with an additional AC-s meter [17]. The measurement was carried out at different depths and covered the whole water column. Additionally, the coefficients $a$ and $c$ were measured at different wavelengths, from $400 \mathrm{~nm}$ to $735 \mathrm{~nm}$. In a scenario where both the receiver and the transmitter are placed at the same depth $d$, the value of $c$ is constant, and the attenuation of a light beam in water can be calculated as (1)

$$
P(r)=P_{0} \cdot e^{-c(d) \cdot r},
$$

where $P(r)$ is the light power propagated to the receiver, assumed to be $r$ meters far from the transmitter. However, in case the transmitter and receiver are located at different depths, respectively $d_{\mathrm{tx}}$ and $d_{\mathrm{rx}}, c$ can vary largely. For this reason, we integrated the depth dependence of the parameter $c$ along the the water column as in (2) below:

$$
P(r)=P_{0} \cdot \prod_{k=K_{0}}^{K_{N}} e^{-c\left(d_{k}\right) \cdot \Delta_{r}\left(d_{k}\right)},
$$

where $d_{K_{0}}=d_{\mathrm{tx}}$ and $d_{K_{N}}=d_{\mathrm{rx}}$, and $\Delta_{r}(d)$ is the distance covered by the beam around depth $d_{k}$ where $c$ can be assumed constant and equal to $c\left(d_{k}\right)$. Moreover, $\sum_{k=K_{0}}^{K_{N}} \Delta_{r}\left(d_{k}\right)=r$. When the CTD was cast, it measured values for $c$ and $d$ along the water column every $30 \mathrm{~cm}$ on average. With this granularity, the variability of two consecutive values of $c$ is always less than $10 \%$, and $0.8 \%$ on average. Hence, it is reasonable to employ linear interpolation to obtain the values of $c(d)$ at depths for which a measurement was not collected. Finally, following the model in [18] and assuming a perfect alignment between the transmitter and the receiver, call $\theta$ the transmitter's beam semi-aperture angle, $A_{r}$ the receiver area and $A_{t}$ the transmitter area. The optical power sensed by the receiver is computed as

$$
P=P(r) \cdot \frac{2 A_{r}}{\pi r^{2}(1-\cos \theta)+2 A_{t}} .
$$

\section{B. Optical noise: solar light noise and temperature gradient}

The downwelling radiance of the sunlight has been measured with the free-falling Satlantic Hyperpro II radiometer [19] along the so-called euphotic zone, while the boat was moving straight at a constant speed. The data was collected at several wavelengths from $400 \mathrm{~nm}$ to $735 \mathrm{~nm}$ throughout the water column. Since the HyperPro measures only the downwelling radiance per unit wavelength $E_{d}$ in $\mathrm{W} /\left(\mathrm{cm}^{2} \mathrm{~nm}\right)$, in the second case we applied a conversion based on the relation between the ratio $E_{0} / E_{d}$ and the ratio between the scattering coefficient and the absorption coefficients $b / a[20$, page 180]. The scattering coefficient has been obtained from the relation $b=c-a$, while the ambient light noise at the receiver is calculated as $N_{A}=E_{0}(d) A_{r} S$.

The CTD also provided measurements of the temperature $T$ of the water throughout the water column. The temperature at the receiver is employed to compute the amount thermal noise affecting the reception, which is related to the shunt resistance, the series resistance and the load resistance: $I_{j}=$ $\sqrt{4 K T B / R}$. Finally, the optical SNR is computed as

$$
S N R=\frac{P \cdot S}{2 q\left(I_{d}+S \cdot P\right) B+4 K T B / R+N_{A}^{2}} .
$$

In order to emphasize how the variation of the sunlight noise irradiance $E_{0}$ and of the total attenuation coefficient $c$ affect optical propagation in a real scenario, we report in Fig. 3 the coverage area of an omni-directional transmitter, represented as a red diamond, placed at a depth of $15 \mathrm{~m}, 45 \mathrm{~m}$ and $75 \mathrm{~m}$ in a water column of about $120 \mathrm{~m}$. The coverage area is defined as the region of the watercolumn where the SNR would be higher than $20 \mathrm{~dB}$. The coverage area is shown both in the presence of sunlight noise (black line) and for night-time operations in dark waters (blue line), when the sunlight noise is considered to be negligible. The results in Fig. 3 highlight the asymmetric shape of the coverage region, and the fact that the region broadens when the transmitter is placed at a deeper location. The latter is due both to the trend followed by $c$, which tends to decrease with depth regardless of the presence of sunlight noise, and due to the lower sunlight noise irradiance.

\section{SimUlation SCENARIO AND SYSTEM CONFIGURATION}

We simulate a scenario akin to those presented in [21] (Fig. 4), where one or more autonomous underwater vehicles (AUVs) perform data muling operations along a network of submerged sensors clusters. A similar scenario is also considered in [22], where the authors focus on optimizing the AUV trajectory to maximize both throughput and power efficiency. In our scenario, each cluster acts as an autonomous data collection entity, and is disconnected from other clusters. The distance between two adjacent clusters is $400 \mathrm{~m}$, each cluster is deployed at different water depths, from 15 to $125 \mathrm{~m}$, and therefore incurs different water conditions. A clusterhead is elected within each cluster to be the only node which can directly talk with a passing AUV.

In this context, acoustic communications are employed to exchange low-rate data when the AUV is cruising from one node to another; high-rate optical communications, instead, support short-distance message transmission at a very high bit rate, and can be exploited whenever the AUV is sufficiently close to a node. A signaling mechanism to automatically detect when this is the case to switch between optical and acoustic communications is employed in the communications stack. Such mechanism has been described first in [23], where we proved the effectiveness of adding a signaling system to a switching strategy based on the perceived power, in a scenario where all the nodes are placed at the same depth and with the same optical channel proprieties.

In our case, a purely threshold-based switching algorithm cannot be employed, due to the high variability of the water conditions. In our solution, the AUV probes the optical channel every time that it approaches a node, and the switch between 

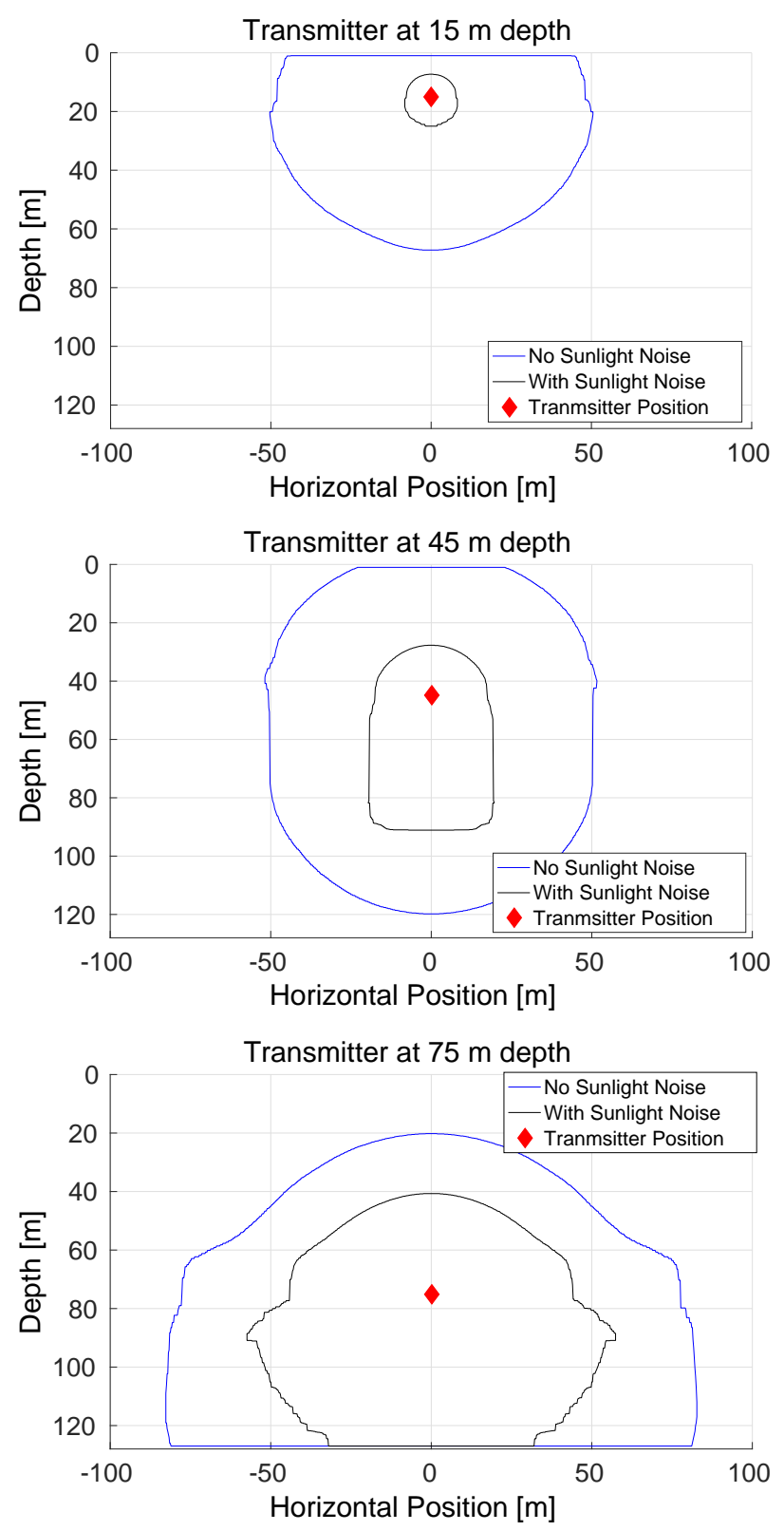

Figure 3. Optical modem coverage area, depending on the transmitter depth. When the transmitter is deeper the region broadens due to a lower $c$ and a lower $E_{0}$ (in the presence of sunlight noise).

acoustic and optical communications occurs only when such probe exchange is successful. To reproduce this behavior, we estimate the distance between the transmitter and the receiver based on the perceived acoustic power: the probing system starts when the estimated distance is smaller than or equal to the maximum optical communication range. On the other end, the AUV switches back to acoustics as soon as the it does not receive any packets for a fixed period of time due to packet loss. Such a system largely fits any applications where the traffic is strongly asymmetric. For example, this is the case when underwater nodes are expected to send large amounts of data to the AUV, whereas the latter only transmits sporadically,

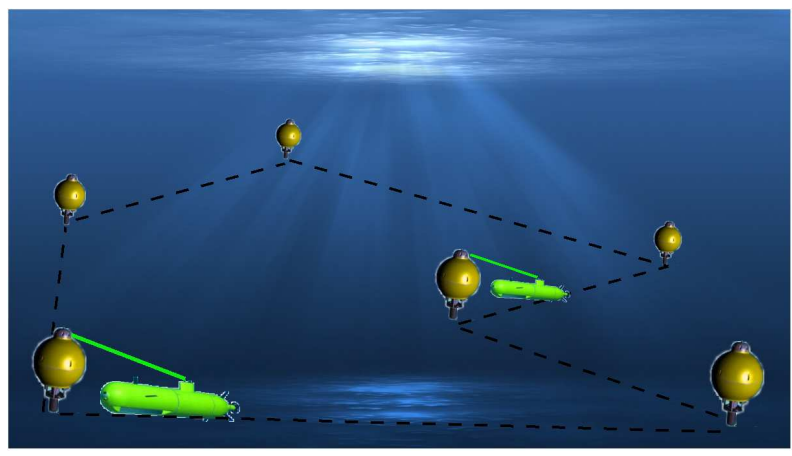

Figure 4. Simulation scenario with two AUVs and six sensor clusters deployed at different depths. The AUVs hover through the network area and collect data from the clusters. The trajectory of the AUVs is shown as a dashed line.

such as in a data-muling application.

In our simulation, the speed of sound under water is assumed to be constant and equal to $1500 \mathrm{~m} / \mathrm{s}$, while the speed of light is set to $\simeq 2.25 \cdot 108 \mathrm{~m} / \mathrm{s}$. We simulate the behavior of the EvoLogics HS acoustic modem [24] for acoustic communications; this modem works at a carrier frequency of 150 $\mathrm{kHz}$ and provides a bandwidth of $60 \mathrm{kHz}$, a nominal maximum transmission rate of $62.5 \mathrm{kbps}$ (as reported in the modem data sheet [24]), a maximum transmission power of $10 \mathrm{~W}$ and a nominal operational SNR of $10 \mathrm{~dB}$. The acoustic bit rate is set to $40 \mathrm{kbps}$, with a source level of $150 \mathrm{~dB}$ re $\mu \mathrm{Pa}$ at $1 \mathrm{~m}$ from the source.

The optical transmission model is implemented according to the description in Section II, assuming an optical wavelength $\lambda=532 \mathrm{~nm}$. The SNR threshold for optical reception is set to $20 \mathrm{~dB}$. The optical transmitter has a transmission rate of 1.5 Mpbs and a transmission power of $100 \mathrm{~W}$. In addition, although our simulator can consider directional beams and non line of sight optical communications as well, we force perfect alignment between transmitter and receiver, which fits well optical transceiver architectures made of multiple emitting and receiving elements arranged, e.g., in a hemispherical topology. The sunlight noise and the temperature gradient have been taken into account as explained in Section II-B. The maximum optical range in such scenario is $62 \mathrm{~m}$, that corresponds to an acoustic threshold of $120 \mathrm{~dB}$ re $\mu \mathrm{Pa}^{2}$ at $1 \mathrm{~m}$ from the source. When the mean received power passes such threshold, we assume that the AUV is approaching a node and the probing mechanism described above begins. The results of such simulations are shown in SectionIV.

\section{RESULTS AND PERFORMANCE COMPARISON}

In order to highlight the benefits of an optical communication simulator based on real channel measurements with high variability of attenuation coefficient and solar noise along the water column, we deploy the nodes at different depths, and set the corresponding AUV waypoints to match such depths. In particular, the northwest node is placed at a depth of $20 \mathrm{~m}$, and the southeast node is placed at a depth of $120 \mathrm{~m}$, whereas the other nodes are placed at intermediate depths: the closer 


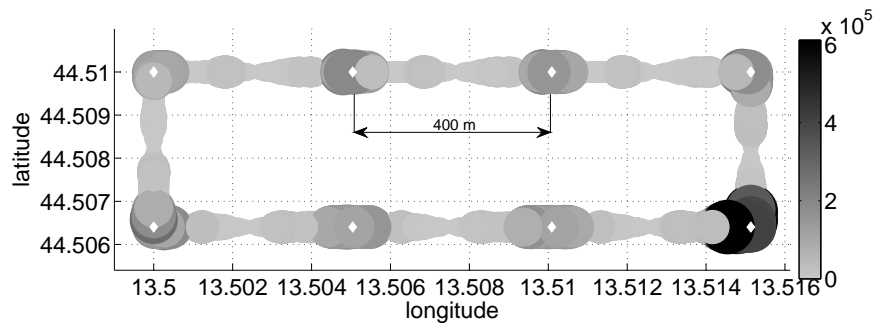

Figure 5. Throughput of the link between the AUV and the node closest to it. The white diamonds represent the node locations.

to the deepest node, the deeper they are placed.

Fig. 5 represents the instantaneous throughput as a function of the AUV position: the thinner the line and the lighter its gray color shade, the lower the throughput. Fig. 5 shows that the switching mechanism described in Section $\mathrm{V}$ is effective: when the AUV approaches a node, the system switches from acoustic to optical communications, and the throughput becomes higher. On the other hand, when the AUV covers the distance from one node to the following one, it switches back to acoustic communications, in order to maintain an active link with the closest node. We can observe a very high throughput close to the deepest node, i.e., the node located at the bottomright position in the figure. This is due to the fact that solar light noise is negligible at a depth of $120 \mathrm{~m}$, and in addition the attenuation coefficient is much smaller than close to the water surface.

Such a behavior is further detailed in Fig. 6, where we report the cumulative distribution function $(\mathrm{CDF})$ of the instantaneous throughput at the receiver, for communications at a depth of 20 to 80 meters from the surface (solid line) and at a depth of 80 to 120 meters (solid line with diamond markers). The difference between the two cases is not very large for a distance above $85 \mathrm{~m}$, where the optical communications cannot be employed; on the contrary, the achievable throughput is much higher in a deep water scenario when the distance decreases below $85 \mathrm{~m}$.

This simulation study suggests that the impact of sunlight noise on the performance of underwater optical communication systems is very large. These results were discussed with the optical communications team of the ENEA Casaccia research center in Italy, that are developing an underwater optical modem prototype for underwater robotic networks. The prototype was tested in a lake experiment in subby shallow waters before proceeding further with the development in order to assess the impact of sunlight noise on the receiver. The next section reports on these experiments and the related conclusions.

\section{REAL MEASUREMENTS OF OPTICAL COMMUNICATION}

We performed a lake test of ENEA's proof-of-concept $(\mathrm{PoC})$ optical modem prototype from October 10 to October 12, 2016 at the Bracciano lake, Italy. The PoC modem has three sides, as seen in Fig. 7, which make it possible to achieve virtually omni-directional communications. The modem can transmit
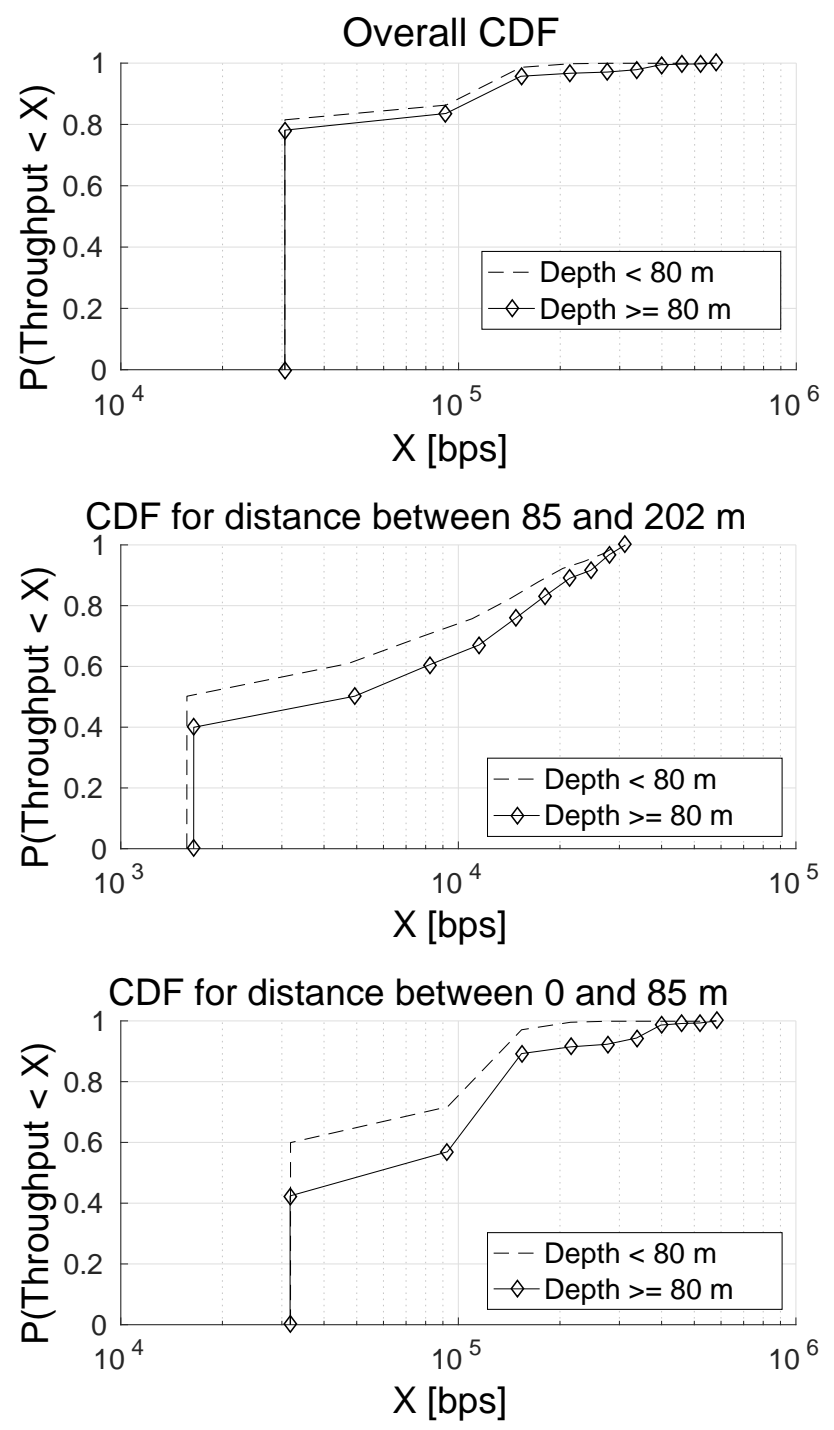

Figure 6. Cumulative distribution function of the throughput between the AUV and the node closest to it.

packets within short ranges, at a bit rate of up to 1 Mbps. The transceiver of the modem has been made using off-theshelf photo-sensors with the human-eye wavelength sensitivity, and blue and white high power LED matrix emitters, usually employed for night lighting. An Arduino Mega board [25] provides the electronics and logic to drive the transceiver. In this preliminary stage, both the transmit power and the receiver-side signal acquisition thresholds are manually set and calibrated. Such calibration was one of the main issues for the experiment, as the external conditions were not stable. The new version of the prototype will employ a self-calibrating system.

The modem was powered with a $48 \mathrm{~V}$ external DC power supply, and the data to be transmitted was transfered from a laptop to the modem via an Universal Serial Bus (USB) cable. The test has been carried out when with a bit rate of at 0.5 Mbps and a packet size of 224 bits. The maximum packet 


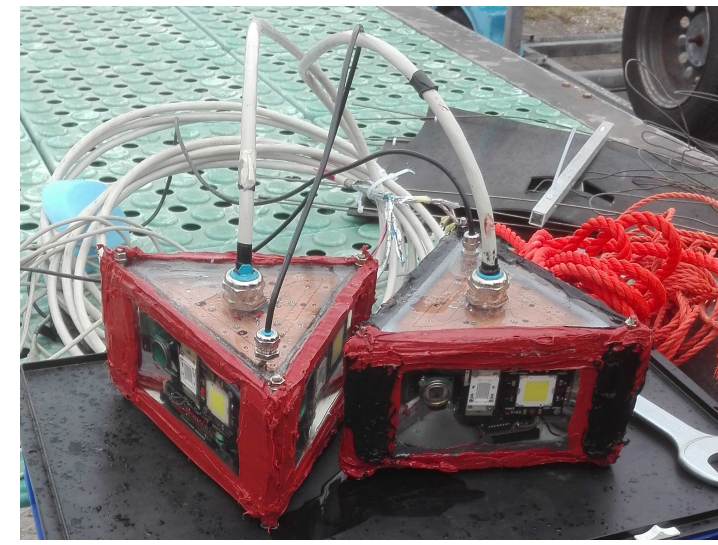

Figure 7. ENEA's proof-of-concept optical modem.

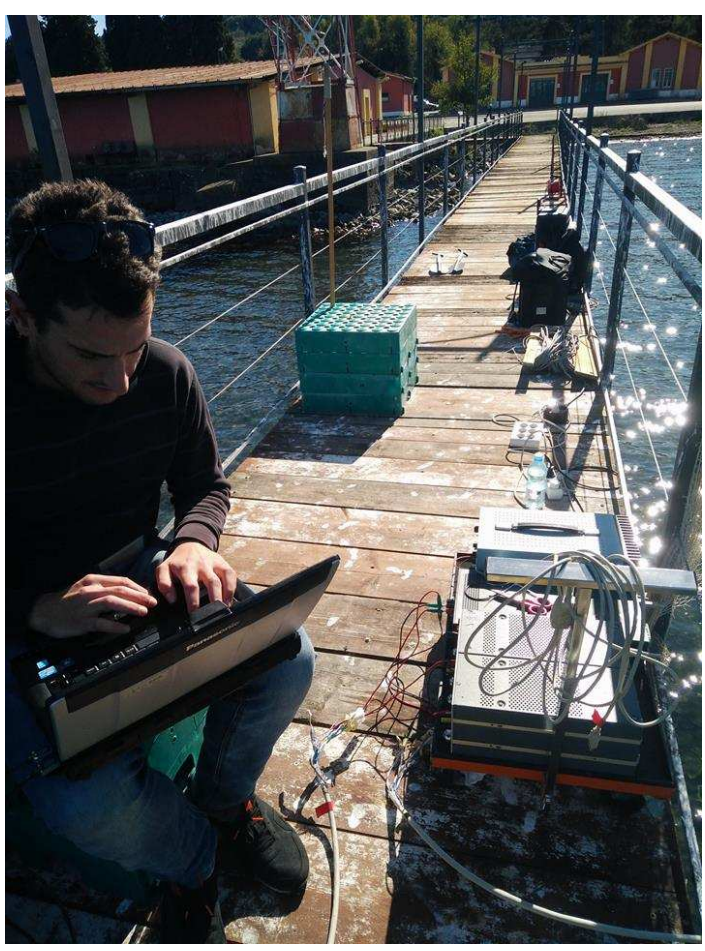

Figure 8. Sunlight conditions during the lake experiment.

size was determined based on the charging time of a capacitor in the low-pass filter employed by the receiver hardware. Such capacitor bacame fully charged a few milliseconds after the reception started, and made it impossible to detect longer packets correctly. This design shortcoming was set to be revised for the next version of the PoC modem.

In our setting, the distance between the transmitter and the receiver was approximately $1.35 \mathrm{~m}$, and the water depth $1.3 \mathrm{~m}$. The water was very clear fresh water, the sky was also clear of clouds and the whole experiment has been carried out around noon in strong sunlight conditions (see Fig. 8).

The DESERT Underwater emulator was employed to perform transmission and reception of the packets via the PoC optical modem. Details and packet delivery ratio (PDR) figures for five different tests carried out during the experiment are reported in the list below.

1) During the fist dry test we performed the transmissions in darker conditions and in the absence of background lighting: this resulted in the successful transmission of $60 \%$ of the packets.

2) A second dry test has been carried out in the presence of blurred background light: in these conditions, the PDR achieved by the modem was around $50 \%$.

3) A final dry test was carried out in the presence of a strong background light, which led to a PDR of 5\%, in addition to an unstable behavior caused by the receiver electornics saturation.

4) A first lake test was carried out by operating the modems in the shade offered by the pier from where the equipment was deployed. The obtained PDR in these conditions was about $10 \%$.

5) A second lake test faced strong sunlight noise, and yielded a PDR of $2 \%$ or less. This figure was partly due to the strong sunlight noise, and partly to the saturation of the receiver.

The PoC version of the ENEA Casaccia modem was not design to withstand the pressure of deeper waters. However, these preliminary results already highlight that the impact of sunlight noise on underwater optical communications cannot be neglected, and possibly requires a carefully designed sunlight noise cancellation mechanism at least to avoid the saturation of the light detector at the receiver. An automatic mechanism to adaptively calibrate the reception threshold would also reduce wrong signal detections and trigger packet reception optimally in different illumination scenarios. Both improvements are under development at ENEA and will be incorporated in the next version of the modem.

\section{CONCLUSIONS}

We presented a measurement-driven optical channel model and its implementation as part of the DESERT Underwater network simulator. The model incorporates samples of three different optical parameter: the temperature, the total attenuation coefficient of the water, and the downwelling solar light irradiance. Such samples have been collected in the context of the NATO STO CMRE ALOMEX'15 scientific cruise. Both parameters are included as part of a depth- and distancedependent signal-to-noise ratio model that can be used to simulate the performance of optical links. Through a simulation study, we proved the model's capability to simulate different water conditions, and depth-dependent optical performance. We verified that the insight provided by the simulator in practice with two proof-of-concept optical modems, which motivated the extension of the modem design to include a solar light filter and automatic receiver threshold settings. Further work will concern a multi-modal optical and acoustic network sea-trial evaluation of our network protocols, such as [26], [27], where both technologies are exploited to optimize the network throughput and minimize the delivery delay. 


\section{ACKNOWLEDGMENT}

This work has been supported in part by the US Office of Naval Research under Grant no. N62909-14-1-N127 and by the European Commission through the 7th Framework Programme, AMAROUT II project, G.A. no. 291803. This work has also received support from the EKOE (Environmental Knowledge and Operation Effectiveness) program of the NATO STO Centre for Maritime Research and Experimentation (CMRE). The dataset was acquired with projectors and hydrophone instruments from the anti-submarine warfare (ASW) program of CMRE. We would like to thank the captain and the crew of NRV Alliance for their kind assistance during data collection operations at sea.

\section{REFERENCES}

[1] "Sonardyne BlueComm Optical Modem ," Accessed: Oct. 2014. [Online]. Available: http://www.sonardyne.com/products/all-products/ instruments/1148-bluecomm-underwater-optical-modem.html

[2] "Neptune underwater optical communications," accessed: Nov. 2016. [Online]. Available: http://www.saphotonics.com/wp-content/uploads/ 2012/08/Neptune-Datasheet.pdf

[3] "Underwater Optical Communications," accessed: Nov. 2016 [Online]. Available: https://www.qinetiq-na.com/products/maritimesys/ underwater-optical-communications/

[4] C. Moriconi et al., "Hybrid acoustic/optic communications in underwater swarms," in Proc. MTS/IEEE OCEANS, Genova, Italy, May 2015.

[5] P. Gois et al., "Development and validation of Blue Ray, an optical modem for the MEDUSA class AUVs," in Proc. Ucomms, Lerici, Italy, Sep. 2016.

[6] D. Anguita et al., "Optical wireless underwater communication for AUV: Preliminary simulation and experimental results," in Proc. IEEE/OES Oceans, Santander, Spain, Jun. 2011.

[7] F. R. Dalgleish et al., "Physical layer simulator for undersea free-space laser communications," Optical Engineering, vol. 53, no. 5, pp. 051410 1027, May 2014.

[8] F. Hanson and S. Radic, "High bandwidth underwater optical communication," Applied Optics, vol. 47, no. 2, pp. 277-283, Jan. 2008.

[9] C. Gabriel et al., "Monte-carlo-based channel characterization for underwater optical communication systems," IEEE/OSA Journal of Optical Communications and Networking, vol. 5, no. 1, pp. 1-12, Jan. 2013.

[10] J. W. N. Farr and, C. Pontbriand, and T. Hammar, "Optical communication system expands cork seafloor observatory's bandwidth," in Proc. MTS/IEEE Oceans, Seattle, US, Sep. 2010.
[11] N. Farr et al., "Optical modem technology for seafloor observatories," in Proc. MTS/IEEE Oceans, Boston, MA, Sep. 2006.

[12] C. Pontbriand et al., "Diffuse high-bandwidth optical communications," in Proc. MTS/IEEE Oceans, Quebec City, Canada, Sep. 2008.

[13] N. Farr et al., "An integrated, underwater optical/acoustic communications system," in Proc. MTS/IEEE Oceans, Sydney, Australia, May 2010.

[14] F. Campagnaro et al., "The DESERT underwater framework v2: Improved capabilities and extension tools," in Proc. Ucomms, Lerici, Italy, Sep. 2016

[15] “S2R-WiSE Series," Last time accessed: February 2014. [Online]. Available: http://www.evologics.de/en/products/developer_tools/index.html

[16] P. Casari et al., "Open-source suites for the underwater networking community: WOSS and DESERT Underwater," IEEE Network, special issue on "Open Source for Networking: Development and Experimentation", vol. 28 , no. 5, pp. 38-46, Sep. 2014

[17] "ac-9 plus," Last time accessed: May 2016. [Online]. Available: http://www.electrotekintl.com/pdf/physical\% 20oceanography/absorption\%20and\%20atteunation\%20meter.pdf

[18] Davide Aguita, Davide Brizzolara, Qilng Hu, "Optical Wireless Underwater Communication for AUV: Preliminary Simulation and Experimental Results," in IEEE Oceans 2011, 6-9 June 2011, pp. 1-5.

[19] "Free-falling optical profiler," Last time accessed: May 2016. [Online]. Available: http://satlantic.com/profiler

[20] J. t.o. Kirk, Light and Photosynthesis in Aquatic Ecosystems. Cambridge University Press, 2007.

[21] I. Vasilescu et al., "Data collection, storage and retrieval with an underwater optical and acoustical sensor network," in Proc. ACM Sensys, San Diego, CA, Nov. 2005.

[22] P. A. Forero et al., "Rollout algorithms for data storage- and energy-aware data retrieval using autonomous underwater vehicles," in Proc. ACM WUWNet, Rome, Italy, Nov. 2014.

[23] F. Campagnaro et al., "Simulation of a multimodal wireless remote control system for underwater vehicles," in Proc. ACM WUWNet, Washington, DC, Oct. 2015.

[24] "Evologics S2C M HS modem," accessed: Feb. 2016. [Online]. Available: http://www.evologics.de/en/products/acoustics/s2cm_hs.html

[25] "Arduino mega 2560," accessed: Mar. 2017. [Online]. Avvailable: https://www.arduino.cc/en/Main/arduinoBoardMega2560

[26] F. Campagnaro et al., "Simulation of multimodal optical and acoustic communications in underwater networks," in Proc. MTS/IEEE OCEANS, Genova, Italy, May 2015.

[27] _ _ "Implementation of a multimodal acoustic-optic underwater network protocol stack," in Proc. MTS/IEEE OCEANS, Shanghai, China, Apr. 2016. 